\title{
Tuberculous Lymphadenitis: Early Diagnosis by GeneXpert of an Auramine-Negative Case.
}

\author{
Louise Munezero ${ }^{*}$, Narcisse Niyikora², Fidèle Mahirane ${ }^{2}$, Belson Rugwizangoga ${ }^{1,2}$. \\ 1 Department of Clinical Biology, School of Medicine and Pharmacy, University of Rwanda, Kigali, Rwanda. \\ 2 Department of Pathology, University Teaching Hospital of Kigali, Kigali, Rwanda
}

*Corresponding author: Louise Munezero. Department of Clinical Biology, School of Medicine and Pharmacy, University of Rwanda, Kigali, Rwanda. Email:louisemunezero113@gmail.com

\begin{abstract}
Tuberculosis (TB) remains one of the major public health problems with a considerable number of new cases. Tuberculous lymphadenitis is one of the most common forms of extra-pulmonary TB whose diagnosis still faces many challenges. The case to report is a 17 -year old female patient with a painful swelling in her right infraretro-auricular area and the auramine stain was negative for acid-fast bacilli (AFB). GeneXpert was done, which confirmed the right infra-retro-auricular tuberculous lymphadenitis. The patient responded well to anti-TB treatment.
\end{abstract}

Key words: Lymphadenitis; Mycobacterium tuberculosis; Auramine; GeneXpert

\section{INTRODUCTION}

Tuberculosis is a chronic granulomatous infection caused by Mycobacterium tuberculosis. It primarily affects the lungs (pulmonary TB, PTB), but more advanced forms of the disease can affect other organs and tissues (extra-pulmonary TB, EPTB).[1] Lymphadenopathy is the most common presentation of extra-pulmonary tuberculosis.[2] Cervical lymph nodes are the most common sites of involvement and reported in $60-90 \%$ of patients with or without involvement of other lymphoid tissue.[3]

In 2017, TB was the ninth leading cause of death worldwide with 6.3 million new cases and estimated 1.3 million TB deaths. EPTB counted for $15 \%$ of the 6.3 million incident cases. [4] The treatment success rate was $82 \%$ meaning that most of TB deaths can be prevented by early diagnosis and appropriate treatment.[5]

Diagnostic and therapeutic challenges consist in that TBL mimics other pathological processes. The World Health Organization (WHO) has recommended the use of GeneXpert for diagnosis of EPTB as the commonly used Auramine stain showed low sensitivity and specificity. However, this recommendation is not yet implemented in Rwanda; the diagnosis of EPTB still relies on auramine or Ziehl-Neelsen stains. This paper reports a case of right infra-retro-auricular tuberculous lymphadenitis that was not detected by auramine stain, but confirmed by GeneXpert.

\section{CASE REPORT}

A 17-year old female patient presented with right infra-retro-auricular swelling for 2 months, associated with intermittent fevers, without cough. She had lost $1 \mathrm{~kg}$ of body weight within that period of time. The physical examination revealed a wellcircumscribed, firm and slightly mobile infra-retroauricular mass of 2-cm diameter (Figure A) and a small cervical lymph node of less than $1-\mathrm{cm}$ diameter at the same side.

Fine Needle Aspiration Cytology (FNAC) was performed and the aspirate was purulent. Diff Quick stain showed polymorphous population of mature and immature lymphocytes (Figure C), aggregates of epithelioid histiocytes (Figure B) and necrosis (Figure D), hence the conclusion of granulomatous lymphadenitis favoring tuberculous lymphadenitis. Auramine stain was negative for AFB. GeneXpert was thus requested on further aspirates, and 
detected $M$. tuberculosis without rifampicin resistance. She was put on anti-TB drugs for 6 months and the patient responded well to the treatment.
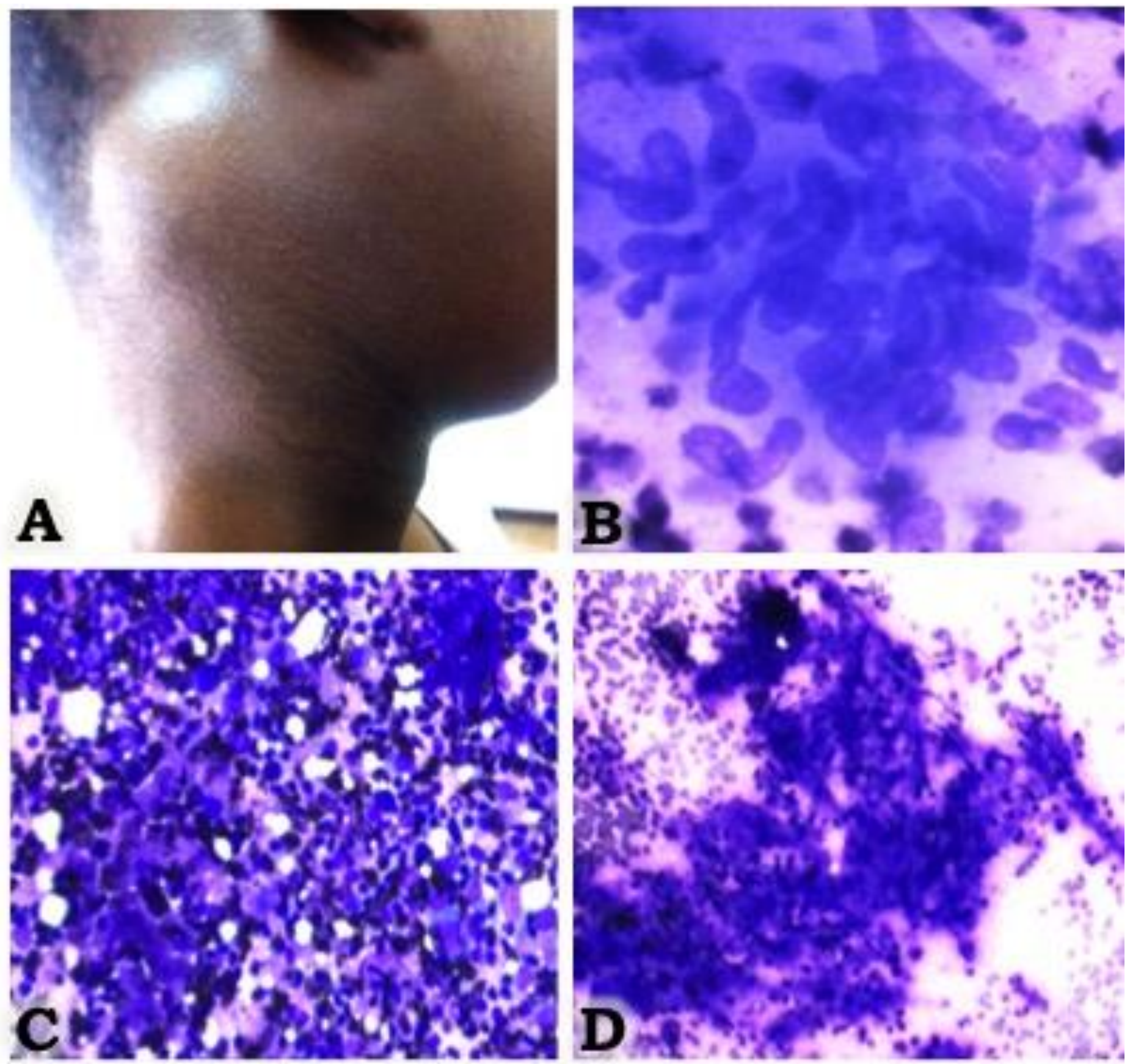

Figure 1. Gross (A) and cytology photomicrographs (B-D) of lymph node tuberculosis: A, Occipital swelling; $\mathrm{B}$, granuloma (H\&E, X400); C, polymorphous population of lymphocytes in necrotic background (H\&E, X100); $\mathrm{D}$, necrosis (H\&E, X100). 


\section{DISCUSSION}

TB possesses a heavy bulk of morbidity amongst the infectious diseases.[6] Clinical manifestations vary, and diagnosis may be challenging. Tuberculous lymphadenitis usually presents as a painless, slowgrowing swelling from a single group of nodes; in $85 \%$ of cases, the involvement is unilateral, the most common being the cervical chain of lymph nodes. [7] The case reported here is relatively uncommon, the swelling was painful and there were infra-retroauricular and cervical lymphadenopathies at the same time.

There are many techniques for $M$. tuberculosis detection which include microscopic examination of Ziehl-Neelsen- or auramine-stained materials, culture and or the newly introduced GeneXpert MTB/RIF for pulmonary and extra-pulmonary samples.[8] Conventional method for AFB detection like auramine stain, generally has a low sensitivity and specificity. GeneXpert has proven to have a high sensitivity and specificity in detecting $M$. tuberculosis and at the same time shows resistant tuberculosis strains from lymph node aspirates.(8)

In this reported case, the auramine stain was negative but GeneXpert was positive and the patient responded well to treatment. This prevented the delay that would have been caused by waiting culture which takes at least two weeks or lymph node excision biopsy for histology, to give the results in 3 weeks or more.[1]

\section{CONCLUSION}

Diagnosis of tuberculous lymphadenitis is still challenging due to the paucibacillary nature of the specimen but also low sensitivity and specificity of some tests which play a role in increasing numbers of false negative cases. GeneXpert implementation in our settings will provide an early and accurate diagnosis in order to decrease TB deaths as there is a good response to the available anti-TB treatment. [5] In addition, GeneXpert can show, at the time of diagnosis, whether or not there is resistance to treatment.

\section{Conflict of interest}

The authors declare that there is no conflict of interest.

This article is published open access under the Creative Commons Attribution-Non Commercial No Derivatives (CC BYNC-ND4.0). People can copy and redistribute the article only for noncommercial purposes and as long as they give appropriate credit to the authors. They cannot distribute any modified material obtained by remixing, transforming or building upon this article. See https://creativecommons.org/licenses/by-nc$\underline{\text { nd } / 4.0 /}$

\section{REFERENCES}

1. Shourya Hegde, K B Rithesh, Kusai Baroudi and DU. Tuberculous Lymphadenitis : Early Diagnosis and Intervention. $\mathrm{J}$ Int oral Heal. 2014;6:96-8.

2. Brijesh Thakur, Ravi Mehrotra and JSN. Correlation of Various Techniques in Diagnosis of Tuberculous Lymphadenitis on Fine Needle Aspiration Cytology. Patholog Res Int. 2013;103.

3. Mulualem Tadesse, Gemeda Abebe, Ketema Abdissa, Dossegnaw Aragaw, Kedir Abdella, Alemayehu Bekele, Mesele Bezabih, Ludwig Apers, Bouke C. de Jong LR. GeneXpert MTB / RIF Assay for the Diagnosis of Tuberculous Lymphadenitis on Concentrated Fine Needle Aspirates in High Tuberculosis Burden Settings. journal.pone. 2015;1-9.

4. World Health Organization (WHO). Global tuberculosis report 2017. 2017.

5. World Health Organization (WHO). Global tuberculosis report 2018. 2018.

6. Yeshi Metaferia, Abdurahaman Seid, Genet Mola Fenta and DG. Assessment of Extrapulmonary Tuberculosis Using Gene Xpert MTB / RIF Assay and Fluorescent Microscopy and Its Risk Factors at Dessie Referral Hospital, Northeast Ethiopia. Biomed Res Int. 2018;10.

7. Guocan Yu, Fangming Zhong, Bo Ye, Xudong Xu, Da Chen and YS. Diagnostic Accuracy of the Xpert MTB/RIF Assay for Lymph Node Tuberculosis: A Systematic Review and MetaAnalysis. Biomed Res Int. 2019;12.

8. Laura Maynard-Smith, Natasha Larke, Jurgens A Peters SDL. Diagnostic accuracy of the Xpert MTB / RIF assay for extrapulmonary and pulmonary tuberculosis when testing nonrespiratory samples: a systematic review. BMC Infect Dis. 2014;14:709. 